\title{
Importancia del estudio de catalizadores para la reducción de compuestos orgánicos de azufre en gasolinas y diésel
}

Importance of the study of catalysts for organosulfur-compounds reduction in gasoline and diesel

\author{
L. Salinas-Palafox (iD a, * \\ a Área Académica de Química, Universidad Autónoma del Estado de Hidalgo, Carretera Pachuca-Tulancingo, Km. 4.5, C.P. 42184, Mineral de la Reforma, Hidalgo, \\ México.
}

\section{Resumen}

Las emisiones de óxidos de azufre $\left(\mathrm{SO}_{\mathrm{x}}\right)$ producidas por la combustión de diésel y gasolina del sector transporte originan contaminación ambiental. Los óxidos de azufre en la atmósfera producen lluvia ácida, que perjudica a los seres vivos y a las edificaciones. Una alternativa para disminuir el contenido de azufre en los combustibles es el proceso de hidrodesulfuración. Este proceso es un tipo de hidrotratamiento que emplea catalizadores para disminuir el contenido de compuestos orgánicos de azufre en gasolinas y diésel. Por lo tanto, los países implementan normas que establecen los límites permitidos para el contenido de azufre en los combustibles, que a veces no se cumplen. Por esta razón, las investigaciones sobre catalizadores en el proceso de hidrodesulfuración continúan vigentes. Este trabajo muestra una revisión de modificaciones realizadas en catalizadores NiW, NiMo y CoMo utilizados en reacciones de hidrodesulfuración y cómo influyen en la actividad catalítica.

\section{Palabras Clave:}

Óxidos de azufre, Hidrodesulfuración, Catalizadores.

\begin{abstract}
Sulfur oxides $\left(\mathrm{SO}_{\mathrm{x}}\right)$ emissions are produced by diesel and gasoline combustions from the transportation sector. Those emissions cause environmental pollution. Sulfur oxides in the atmosphere produce acid rain, which harms living beings and buildings. An alternative to decrease the sulfur content in fuels is the hydrodesulfurization process. This process is a catalytic hydrotreating for decreasing the content of organo-sulfur compounds. Therefore, environmental regulations have been implemented to regulate the limits of sulfur in fuels, but sometimes, they do not fulfill those regulations. For this reason, the research on catalysts continues. This work reviews the state of art about NiW, NiMo and CoMo catalysts used in hydrodesulfurization reactions and how their modifications influence the catalytic activity.
\end{abstract}

Keywords:

Sulfur oxides, Hydrodesulfurization, Catalysts.

\section{Introducción}

Los óxidos de azufre $\left(\mathrm{SO}_{\mathrm{x}}\right)$ se producen durante la combustión de la gasolina y el diésel. Estos óxidos dañan a los seres vivos y a los materiales de las edificaciones. Los $\mathrm{SO}_{\mathrm{x}}$ interaccionan con el agua de lluvia para producir ácido sulfúrico $\left(\mathrm{H}_{2} \mathrm{SO}_{4}\right)$ en la conocida lluvia ácida, la cual ocasiona la corrosión de $\mathrm{Fe}, \mathrm{Zn}$ y acero, además de dañar los materiales de construcción carbonatados como el mármol y las calizas (Puigcerver and Carrascal, 2008). La lluvia ácida contribuye a acidificar los ríos, los lagos y los mares modificando el desarrollo de la vida en este tipo de ecosistemas. Además, esta precipitación perturba la cadena alimentaria y disminuye el $\mathrm{pH}$ de los suelos carentes de carbonato de calcio $\left(\mathrm{CaCO}_{3}\right)$. Cuando el pH de éstos suelos es cercano a 4, el hidróxido de aluminio $\left(\mathrm{Al}(\mathrm{OH})_{3}\right)$ presente, se disuelve, promoviendo su absorción de los cultivos. Además, se transporta a las aguas, originando el envenenamiento de los peces (Castillo et al., 2005).

La problemática del contenido de $\mathrm{SO}_{2}$ a nivel mundial, ha requerido realizar monitoreos atmosféricos de partículas nocivas para el medio ambiente. Copérnico es un Programa de Observación de la Tierra de la Unión Europea que muestra datos de monitoreo atmosférico a nivel mundial de contaminantes como el monóxido de carbono (CO), dióxido de

*Autor para la correspondencia: lilisalinasp@gmail.com

Correo electrónico: lilisalinasp@gmail.com (Liliana Salinas-Palafox) 
carbono $\left(\mathrm{CO}_{2}\right)$, metano $\left(\mathrm{CH}_{4}\right)$, dióxido de nitrógeno $\left(\mathrm{NO}_{2}\right)$, dióxido de azufre $\left(\mathrm{SO}_{2}\right)$, etc., con el fin de gestionar de manera sostenible el ambiente que habitamos.

Un mapeo del monitoreo de $\mathrm{SO}_{2}$ a nivel mundial muestra más de $1 \times 10^{15}$ moléculas de $\mathrm{SO}_{2}$ por $\mathrm{cm}^{2}$ en prácticamente todo el mundo (indicando aumento de concentración desde el color azul hacia el rojo), Figura 1.

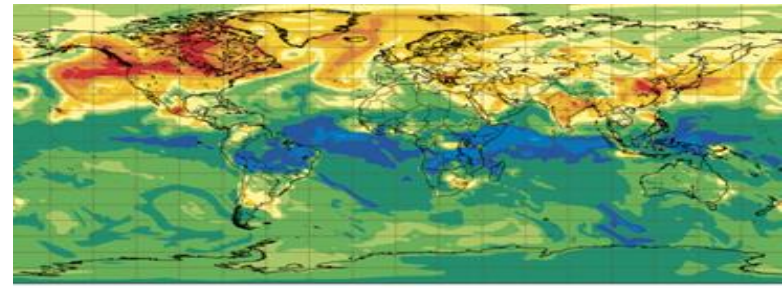

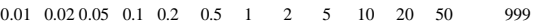

Columna de moléculas totales de dióxido de azufre $\left(\mathrm{x} 10^{15}\right.$ moléculas de $\mathrm{SO}_{2}$ por $\left.\mathrm{cm}^{2}\right)$

Figura 1. Contenido de moléculas de $\mathrm{SO}_{2}$, desde $\mathrm{0.01}$ (color azul) hasta $999 \times 10^{15}$ (color rojo) por $\mathrm{cm}^{2}$. Proporcionado por el CAMS, el servicio de monitoreo atmosférico Copérnico [2020]. Mapa tomado el 22 de marzo de 2020.

El centro de México presenta la mayor afectación por las emisiones de $\mathrm{SO}_{2}$, con hasta $999 \times 10^{15}$ moléculas de $\mathrm{SO}_{2}$ por $\mathrm{cm}^{2}$. Lo cual corresponde a $0.1 \mathrm{mg}$ de $\mathrm{SO}_{2}$ por $\mathrm{cm}^{2}$. Además de encontrarse en las zonas más contaminadas en el mundo por partículas de $\mathrm{SO}_{2}$ (indicado en color rojo), Figura 2.

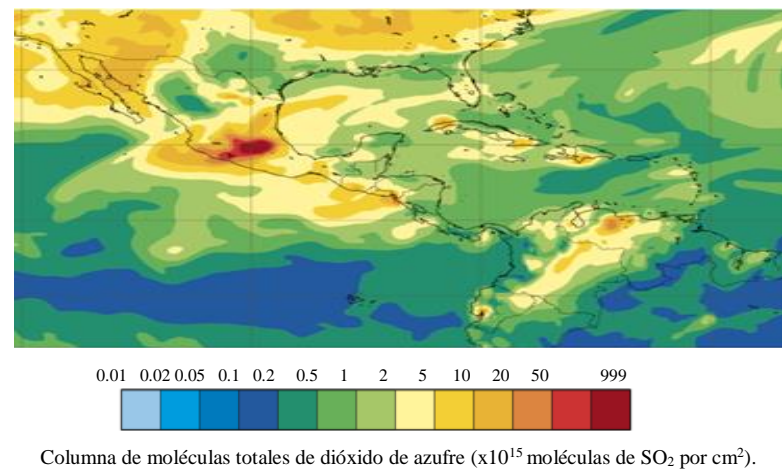

Figura 2. Contenido de moléculas de $\mathrm{SO}_{2}$ en Centroamérica, desde 0.01 hasta $999 \times 10^{15}$ por $\mathrm{cm}^{2}$, proporcionado por CAMS, el servicio de monitoreo atmosférico Copérnico [2020]. Mapa tomado el 22 de marzo de 2020 .

Esta cifra es alarmante debido a que en los seres humanos, las emisiones de $\mathrm{SO}_{\mathrm{x}}$ de hasta $25 \mathrm{mg} / \mathrm{kg}$ de aire afectan a la parte alta del sistema respiratorio. Mientras que una exposición prolongada podría originar problemas respiratorios.

Además, el uso de combustibles con alto contenido de azufre disminuye la eficiencia de los convertidores catalíticos en los vehículos automotrices, evitando que se lleven a cabo las reacciones catalíticas de interés. El contenido de azufre, por lo tanto, impacta en los costos de las gasolinas, siendo la gasolina Magna la que contiene mayor cantidad de azufre.

Los problemas ambientales ocasionados por los óxidos de azufre han llevado a proponer regulaciones sobre el contenido de azufre en los combustibles como el diésel y la gasolina. En México, la Norma Oficial Mexicana (D.O.F., 2006) establece un máximo de $15 \mathrm{mg} / \mathrm{kg}$ de azufre en diésel y un máximo de $80 \mathrm{mg} / \mathrm{kg}$ de azufre en gasolinas desde el año 2009. Sin embargo, el diésel y las gasolinas comerciales a veces no lo cumplen.

Una estrategia para atender este tipo de problemas ambientales es mejorar la calidad de los combustibles. Lo cual se puede realizar a través de procesos catalíticos de hidrotratamiento, particularmente el proceso de hidrodesulfuración. Esta es la razón por la que se investigan los catalizadores desde los procesos de impregnación hasta su activación.

\section{Hidrotratamiento}

El hidrotratamiento es utilizado ampliamente en la conversión de corrientes de crudos de petróleo y en la mejora de la calidad de los productos finales, para controlar el contenido de compuestos aromáticos, $\mathrm{S}, \mathrm{N}, \mathrm{O}$ y metales así como el número de octano/cetano.

En este proceso, una hidrogenación catalítica se realiza a altas presiones de $\mathrm{H}_{2}$, en la cual los hidrocarburos insaturados se isomerizan o se saturan. También durante este proceso ocurre la remoción de $\mathrm{S}, \mathrm{N}, \mathrm{O}$ y metales de las corrientes de petróleo en la refinería.

En la industria de la refinación del petróleo, los procesos de hidrotratamiento se clasifican de acuerdo con la severidad de la operación. El tamaño molecular no cambia esencialmente bajo condiciones relativamente suaves de operación (Topsøe et al., 1996). Es decir, las condiciones de hidrotratamiento saturan de hidrógeno los compuestos de la corriente en la refinería y/o remueven heteroátomos indeseados del petróleo sin cambiar significativamente el rango del punto de ebullición o la distribución del tamaño molecular.

Los crudos del petróleo se componen, en su mayoría, de hidrocarburos, y dependiendo de su origen, varía la cantidad de átomos de $\mathrm{S}, \mathrm{N}, \mathrm{O}$ y metales. El contenido de azufre tiene influencia en el valor del crudo de petróleo puesto que un mayor contenido en peso de azufre requiere un proceso más extenso. La desulfuración profunda de las corrientes de petróleo se realiza cuando las condiciones de presión y de temperatura de hidrodesulfuración incrementan. Desafortunadamente, estas condiciones generan reacciones indeseadas como la saturación de olefinas y la disminución del número de octano. El proceso a alta temperatura permite incrementar la formación de coque y la subsecuente desactivación del catalizador. Por esta razón, es importante tomar en cuenta que la severidad de las operaciones está limitada por el diseño de la unidad de hidrodesulfuración. En vez de aplicar estas condiciones, los catalizadores para hidrodesulfuración pueden ser mejorados en actividad (Babich and Moulijn, 2003).

Debido a las normas ambientales relacionadas a las emisiones de contaminantes, el interés en temas relacionados sobre catálisis para el proceso de hidrodesulfuración ha aumentado en los últimos años.

\section{Hidrodesulfuración}

El proceso de hidrodesulfuración (HDS) es un tipo de hidrotratamiento que permite eliminar el azufre presente en las fracciones de petróleo utilizando $\mathrm{H}_{2}$ y un catalizador (Ho, 2004). El azufre se elimina en forma de $\mathrm{H}_{2} \mathrm{~S}$, Figura 3. 


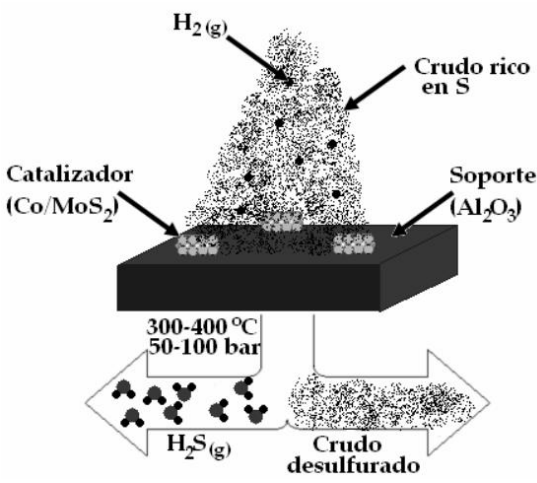

Figura 3. Ilustración del proceso de hidrodesulfuración (Lizama, 2009).

En la refinación del petróleo crudo, éste se somete a una presión aproximada de 1 atmósfera y los componentes se separan con respecto a su punto de ebullición. Con este proceso se obtienen las siguientes fracciones del petróleo crudo, ordenados de menor a mayor punto de ebullición: gas licuado de petróleo y gas, gasolina de destilación directa, nafta, medios destilados, gasóleos pesados y finalmente los residuos. Los residuos son procesados en la sección de destilación al vacío donde se obtiene el gasóleo de vacío y el residuo de vacío.

Los hidrocarburos provenientes de la destilación primaria que se someten a la desulfuración son la gasolina de destilación directa, la nafta, los medios destilados y el gasóleo pesado. Asimismo, tanto el gasóleo pesado como el gasóleo de vacío se someten a una desulfuración para transformarlos en la unidad de craqueo de lecho fluidizado (FCC, por sus siglas en inglés) para obtener nafta FCC y aceite de ciclo ligero, estos dos productos se someten a una desulfuración. Esto para mejorar la calidad de los productos como su número de octano/cetano y reducir los contaminantes después de su combustión. De esta manera, se obtienen gas combustible de refinería, gasolina, keroseno, diésel, aceite de calefacción y combustibles industriales, Figura 4.

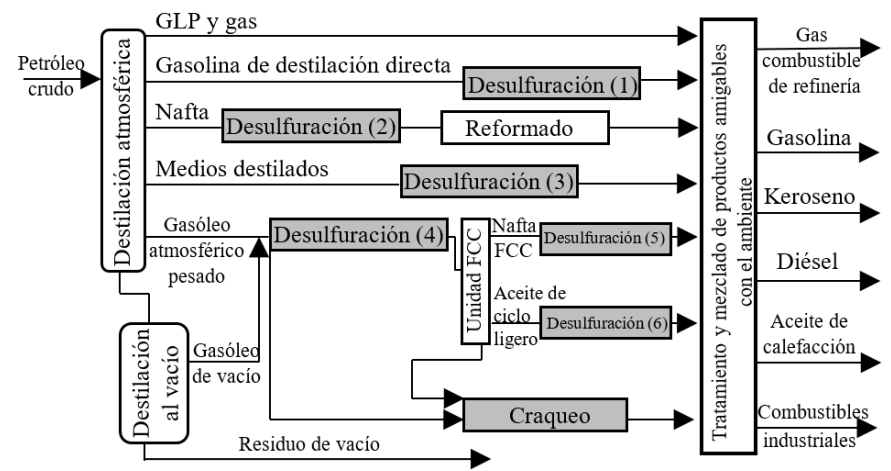

Figura 4. Esquema simplificado de una refinería de petróleo con las posibles ubicaciones de las unidades de hidrotratamiento (Babich and Moulijn, 2003).

Los destilados de petróleo contienen alrededor de 1 a $3 \%$ $\mathrm{p} / \mathrm{p}$ de contenido de azufre. Este azufre se presenta en forma de compuestos orgánicos de azufre, como el tiofeno, benzotiofeno, dibenzotiofeno y alquildibenzotiofeno. Los compuestos con azufre generalmente aumentan de concentración en fracciones con punto de ebullición más alto y en los residuos no volátiles. En la fracción de nafta de bajo punto de ebullición, el azufre se encuentra presente principalmente en forma de tioles, sulfuros, disulfuros o tiofenos. Para las fracciones de medios destilados están presentes los benzotiofenos y dibenzotiofenos. El contenido total de aromáticos incrementa con el aumento del punto de ebullición de la fracción. Las técnicas analíticas como la cromatografía de líquidos de alta resolución (HPLC), cromatografía de gases acoplada a masas (GC-MS) y la resonancia magnética nuclear del isótopo de ${ }^{13} \mathrm{C}\left({ }^{13} \mathrm{C}-\mathrm{NMR}\right)$ han proporcionado información de los compuestos aromáticos en las fracciones de los crudos de petróleo (Stanislaus and Cooper, 1994), (Wilson et al., 1985).

Las velocidades de desulfuración de los compuestos azufrados han sido reportadas (Nag et al., 1979) de la siguiente manera: tiofeno > benzotiofeno $>$ dibenzotiofeno. Esta diferencia de reactividad depende del tamaño molecular y de la estructura del compuesto que contenga el azufre. El tiofeno, el benzotiofeno, el dibenzotiofeno y el alquildibenzotiofeno se han utilizado como moléculas modelo para estudiar la reacción de hidrodesulfuración, siendo más fácil de desulfurar el tiofeno. Mientras que es más difícil desulfurar el alquildibenzotiofeno.

\section{Catalizadores heterogéneos}

Los catalizadores heterogéneos generalmente se componen de uno o varios metales soportados en un sólido. Este tipo de catalizadores presentan las ventajas con respecto a los catalizadores homogéneos de una separación del catalizador de los productos de reacción, sencilla y económica (Shriver et al., 2004), la estabilidad térmica y la regeneración catalítica (Topsøe et al., 1996). No obstante, la desventaja de los catalizadores heterogéneos es la dificultad para realizar su caracterización debido a que sus sitios activos no son uniformes.

Los catalizadores de CoMo, NiMo y NiW con concentraciones aproximadas de $1-4 \% \mathrm{p} / \mathrm{p}$ de $\mathrm{Co}$ o Ni, $8-16 \%$ $\mathrm{p} / \mathrm{p}$ de Mo y de 12-25\% p/p de $\mathrm{W}$ son utilizados para el hidrotratamiento (Topsøe et al., 1996). Los catalizadores de CoMo son utilizados para hidrodesulfuración, por lo tanto, consumen poco $\mathrm{H}_{2}$. Los catalizadores de NiMo son más eficientes para reacciones de hidrogenación, por lo que su consumo de $\mathrm{H}_{2}$ es alto. Los catalizadores de NiW presentan mayor actividad de hidrogenación aromática y procesos de hidrocraqueo (HCR) (Topsøe et al., 1996). Estos catalizadores se han estudiado en reacciones de hidrodesulfuración, a pesar de sus usos particulares, Tabla 1.

Tabla 1: Catalizadores utilizados en procesos de hidrotratamiento

\begin{tabular}{cc}
\hline Catalizador & Uso \\
\hline CoMo & $\begin{array}{r}\text { Hidrodesulfuración de corrientes de } \\
\text { hidrocarburos insaturados }\end{array}$ \\
NiMo & $\begin{array}{c}\text { En corrientes que necesitan hidrogenación } \\
\text { extensiva }\end{array}$ \\
NiW & Hidrogenación aromática e hidrocraqueo \\
\hline
\end{tabular}

Este tipo de catalizadores soportados consisten en una fase activa que es la responsable de la actividad catalítica, un promotor que mejora las propiedades de la fase activa y un soporte donde se depositan los precursores del catalizador (Topsøe et al., 1996) 


\section{Promotor}

Un promotor es un metal que se le agrega al catalizador para estabilizar la estructura de la fase activa, que es la responsable de la velocidad de reacción. Además puede aumentar la selectividad o la resistencia a la desactivación del catalizador. $\mathrm{El} \mathrm{Ni}$ es un promotor en catalizadores que contiene W. Mientras que el Co se utiliza como promotor tanto en catalizadores que contienen $\mathrm{W}$ como en los que contienen Mo. Generalmente, la relación molar entre el promotor $(\mathrm{Co}$ o $\mathrm{Ni}$ ) y el metal (W o Mo) es menor a 0.4 (Ho, 2004). Sin embargo, este valor se puede modificar por la variación de los soportes.

Existe una relación molar óptima promotor/promotor + $\mathrm{W}(\mathrm{Mo})$ que depende de la concentración total de los sitios del borde de sulfuro de tungsteno $\left(\mathrm{WS}_{2}\right)$ que son necesarios para lograr la fase activa y para que se enlacen los átomos de promotor. Cuando el promotor no interactúa con el Mo o el W, se puede enlazar con el soporte para formar especies que son difíciles de activar por sulfuración. Las cuales disminuyen el efecto promotor en la fase activa del catalizador. Por esta razón, la cantidad óptima de promotor y las técnicas en que permanezca la interacción promotor-metal en la superficie han sido estudiadas.

\section{Fase activa}

Los catalizadores para hidrodesulfuración tienen una fase activa que consiste en sulfuros de metales de transición. La reacción de hidrodesulfuración utiliza sulfuros de molibdeno $\left(\mathrm{MoS}_{2}\right)$ o de tungsteno $\left(\mathrm{WS}_{2}\right)$ soportados normalmente en gamma-alúmina $\left(\gamma-\mathrm{Al}_{2} \mathrm{O}_{3}\right)$ y promovidos por $\mathrm{Ni}$ o $\mathrm{Co}$. Con el fin de obtener la fase activa, los óxidos metálicos soportados se activan por sulfuración con una mezcla de $\mathrm{H}_{2} \mathrm{~S} / \mathrm{H}_{2}$. El modelo de Topsøe (Topsøe, 2007), es el más aceptado para explicar la actividad catalítica.

Las fases activas formadas por el metal (W o Mo), el promotor ( $\mathrm{Ni}$ o $\mathrm{Co})$ y el azufre son las responsables de la actividad catalítica. Estas fases se nombran como CoMoS, NiWS, NiMoS, según corresponda. La formación de fases activas depende de la preparación y la activación del catalizador. Esta es la razón por la que es importante estudiar el tipo de óxidos que se depositan en la superficie del catalizador. Además de analizar el efecto que tienen en la actividad catalítica.

La fase CoMoS (NiWS, NiMoS) involucra láminas simples o múltiples de los precursores sulfurados. Las láminas formadas son del tipo I o del tipo II. Las estructuras tipo I son monocapas de $\mathrm{WS}_{2}\left(\mathrm{MoS}_{2}\right)$ que se enlazan con el soporte generando enlaces metal-O-soporte a través de los grupos $\mathrm{OH}-$ durante la preparación del catalizador, estas estructuras son menos activas que las tipo II (Hinnemann et al., 2005) por la menor cantidad de sitios activos disponibles. Las estructuras tipo II están formadas por multicapas de $\mathrm{WS}_{2}\left(\mathrm{MoS}_{2}\right)$, las cuales interactúan débilmente con el soporte y poseen mayor cantidad de sitios activos disponibles.

Las láminas que se forman al sulfurar los óxidos metálicos poseen forma hexagonal con bordes intercalados, un borde de Mo (W) y de azufre y otro borde de azufre con promotor. Cerca del borde de azufre, se encuentran los sitios denominados BRIM que también contienen azufre y poseen carácter metálico. La lámina ubicada en la parte superior de un apilamiento es la que posee más sitios BRIM disponibles y por lo tanto, mayor número de sitios donde la molécula puede adsorberse para que ocurra la reacción catalítica.

Topsøe y colaboradores (Wivel et al., 1981) sugieren que después de la activación del catalizador por sulfuración, algunos átomos del promotor pueden formar sulfuros sobre el soporte, formando algunas fases como los sulfuros de cobalto o sulfuros de níquel $\left(\mathrm{Cog}_{9} \mathrm{~S}_{8}\right.$ o $\left.\mathrm{NiS}_{\mathrm{x}}\right)$, las cuales deben evitarse, ya que no forman parte de las fases activas CoMoS (NiWS, NiMoS).

La fase activa puede actuar de la siguiente manera: La molécula a desulfurar se puede adsorber en los sitios BRIM. El hidrógeno de los sitios de borde vecinos en forma de $-\mathrm{SH}$ se puede transferir a la molécula. Después, el anillo se puede romper e hidrogenarse y posteriormente, desorberse (Topsøe et al., 1996). Asimismo, el azufre se puede desorber en forma de ácido sulfhídrico $\left(\mathrm{H}_{2} \mathrm{~S}\right)$.

\section{Soporte}

El soporte es un material poroso, en el cual se deposita y se dispersa la fase activa y provee la estabilidad térmica y mecánica al catalizador. Los soportes generalmente utilizados en procesos de hidrotratamiento son los de óxido de aluminio $\left(\mathrm{Al}_{2} \mathrm{O}_{3}\right)$, dióxido de silicio-óxido de aluminio $\left(\mathrm{SiO}_{2}-\mathrm{Al}_{2} \mathrm{O}_{3}\right)$, dióxido de silicio $\left(\mathrm{SiO}_{2}\right)$, dióxido de titanio $\left(\mathrm{TiO}_{2}\right)$ y zeolitas. Estos soportes poseen áreas superficiales que van desde los 100 hasta $300 \mathrm{~m}^{2} / \mathrm{g}$. El tamaño y la forma del poro son factores a investigar ya que puede haber obstrucción por deposición de metales o formación de coque (Topsøe et al., 1996).

El óxido de aluminio $\left(\mathrm{Al}_{2} \mathrm{O}_{3}\right)$ es el soporte más utilizado en catalizadores industriales por sus propiedades texturales, mecánicas y su costo. El soporte de $\gamma-\mathrm{Al}_{2} \mathrm{O}_{3}$ industrial tiene de área superficial $270 \mathrm{~m}^{2} / \mathrm{g}$ (Zuo et al., 2004b). Los catalizadores generalmente se soportan en $\mathrm{Al}_{2} \mathrm{O}_{3}$ y se le realizan modificaciones adicionando otros óxidos.

\section{Catalizadores NiW}

Los sulfuros de NiW son utilizados menos frecuentemente en las reacciones de hidrodesulfuración debido a sus costos (Guisnet and Gilson, 2002).

La adición de $\mathrm{Ni}$ en catalizadores $\mathrm{NiW}$ soportados en $\mathrm{Al}_{2} \mathrm{O}_{3}$ para hidrodesulfuración de tiofeno, aumenta aproximadamente 30 veces la velocidad de reacción en tiofeno en comparación a un catalizador de $\mathrm{W}$ soportado en $\mathrm{Al}_{2} \mathrm{O}_{3}$. Además, al agregar $\mathrm{Ni}$ a los catalizadores de $\mathrm{W}$, se mejora la dispersión del $\mathrm{WS}_{2}$ en la superficie (Zuo et al., 2004b). El incremento de la concentración del promotor incrementa la actividad catalítica. Este incremento pasa a un máximo con relaciones molares $\mathrm{Ni} /(\mathrm{Ni}+\mathrm{W})$ de 0.3 a 0.5 (Topsøe et al., 1996).

Investigaciones han mostrado que las modificaciones del soporte y los precursores del catalizador influyen en la actividad con diferentes moléculas modelo. Además, el uso de agentes quelantes permiten que las especies de $\mathrm{Ni} \mathrm{y} \mathrm{W}$ tengan una mejor sulfuración para formar las fases activas, ya que las especies de $\mathrm{Ni}$ y $\mathrm{W}$ se sulfuran a diferente temperatura (Scheffer et al., 1990). La hidrodesulfuración de molécula refractaria, el 4,6-dimetidibenzotiofeno, requirió una relación molar Ni/W de 0.55 utilizando EDTA como agente quelante en catalizadores NiW soportados en zeolitas (Zhang et al., 
2018). Mientras que en reacciones de hidrodesulfuración con tiofeno, que es la molécula modelo menos impedida estéricamente, usando un catalizador $\mathrm{NiW}$ soportado en $\mathrm{Al}_{2} \mathrm{O}_{3}$ la relación óptima de $\mathrm{Ni} / \mathrm{Ni}+\mathrm{W}$ fue de 0.41 (Zuo et al., 2004a).

Otras investigaciones han reportado que la actividad de catalizadores para hidrodesulfuración puede ser debida a la presencia de politungstatos formados desde la síntesis del catalizador (Mogica-Betancourt et al., 2014). Por lo tanto, las investigaciones sobre las estructuras responsables de la formación de fases activas NiWS se deben realizar desde la síntesis del catalizador.

\section{Catalizadores NiMo}

Los catalizadores NiMo poseen una alta actividad de hidrogenación. Por esta razón, estos catalizadores se prefieren para hidrodesulfurar corrientes que necesitan una hidrogenación extensiva. La ventaja de utilizar catalizadores de Mo promovidos por $\mathrm{Ni}$ es que la cantidad requerida de promotor es menor que en catalizadores NiW. Investigaciones indican que la relación molar óptima de $\mathrm{Ni} / \mathrm{Mo}$ es 0.3 para hidrodesulfuración de tiofeno en catalizadores soportados en $\mathrm{Al}_{2} \mathrm{O}_{3}$ (Vissenberg et al., 2001). Además de que es más fácil de sulfurar que el W (Hensen, 2007).

Algunos cambios a este tipo de catalizadores se han realizado modificando el soporte de $\mathrm{Al}_{2} \mathrm{O}_{3}$ con el uso de soportes ácidos adicionando dióxido de titanio $\left(\mathrm{TiO}_{2}\right)$ y el uso de soportes básicos a los que se les adiciona óxido de magnesio $(\mathrm{MgO})$. El uso de soportes básicos ha mostrado una mejora en la dispersión del Mo en la superficie del catalizador para la hidrodesulfuración de dibenzotiofeno (Chary et al., 1991).

\section{Catalizadores CoMo}

Este tipo de catalizadores son los que realizan la desulfuración con un consumo de $\mathrm{H}_{2}$ bajo. Esta característica hace a este tipo de catalizadores atractivos para utilizarse en corrientes de hidrocarburos insaturados.

La relación molar óptima de $\mathrm{Co} / \mathrm{Mo}$ es 0.5 para la hidrodesulfuración de tiofeno en catalizadores soportados en $\mathrm{Al}_{2} \mathrm{O}_{3}$ (Vissenberg et al., 2001). Es decir, se utiliza mayor cantidad de promotor en catalizadores CoMo que en los catalizadores NiMo.

Otro tipo de investigaciones realizadas a los catalizadores CoMo soportados en $\mathrm{Al}_{2} \mathrm{O}_{3}$ son la adición de ácido fosfórico $\left(\mathrm{H}_{3} \mathrm{PO}_{4}\right)$ en la solución de impregnación, lo cual mejora la actividad de hidrodesulfuración de dibenzotiofeno (Vatutina et al., 2019).

Los catalizadores CoW no se discuten en este trabajo ya que se ha encontrado que no existe un efecto promotor entre el Co y el W (Vissenberg et al., 2001).

\section{Conclusiones}

Las investigaciones en reacciones de hidrodesulfuración muestran los efectos de las modificaciones en los catalizadores de Mo o W. Las modificaciones han incluido el uso de aditivos en la síntesis del catalizador como la adición de $\mathrm{H}_{3} \mathrm{PO}_{4} \mathrm{o}$ de agentes quelantes, el estudio de los precursores en estado óxido, la modificación de los soportes y la cantidad óptima de promotor.
El uso de aditivos como el $\mathrm{H}_{3} \mathrm{PO}_{4}$ ha mostrado tener efectos benéficos en la actividad catalítica. La presencia de agentes quelantes permite mejorar el efecto promotor. El cambio del soporte modifica la manera en que se dispersa la fase activa en la superficie. Además la optimización de un catalizador debe considerar el tipo de molécula modelo a estudiar.

Si bien el objetivo es estudiar catalizadores más activos para los procesos de hidrodesulfuración, para poder hacer una comparación entre cuál catalizador sería más factible, se deberían homologar condiciones. Esto es debido a que se estudian a diferentes condiciones de presión, de temperatura, de moléculas modelo, de concentración de los metales, de soportes, de aditivos, etc. Las normas ambientales son una de las justificaciones de este tipo de investigaciones. Lo que muestra este trabajo son las diferencias y las modificaciones entre los catalizadores más comunes como los CoMo, NiMo y NiW.

Estos son algunos de los parámetros que se pueden manipular para cambiar la funcionalidad de un catalizador para alcanzar una desulfuración profunda como lo piden las normas ambientales.

\section{Agradecimientos}

Este trabajo ha sido realizado gracias al apoyo de una beca de maestría por parte del Consejo Nacional de Ciencia y Tecnología (CONACYT).

\section{Referencias}

Castillo, R. F., Roldán, R. M. D., Blasco, P. R., Huertas, R. M. J, Caballero, D. F. J., Moreno, V. C., Luque, R. M., 2005. Biotecnología Ambiental. Tébar, Madrid.

Babich, I., Moulijn, J.A., 2003. Science and technology of novel processes for deep desulfurization of oil refinery streams: a review. Fuel 82, 607-631. DOI:10.1016/s0016-2361(02)00324-1

Chary, K. V. R., Ramakrishna, H., Rama Rao, K. S., Murali Dhar, G., Kanta Rao, P., 1991. Hydrodesulfurization on $\mathrm{MoS}_{2} / \mathrm{MgO}$. Catalysis Letters 10, 27-33. DOI:10.1007/bf00764733

D. O. F., 2006. NOM-086-SEMARNAT-SENER-SCFI-2005. Especificaciones de los combustibles fósiles para la protección ambiental. Diario Oficial de la Federación: Ciudad de México, México.

Guisnet, M., Gilson, J. P., 2002. Zeolites for Cleaner Technologies. Imperial College Press, Londres.

Hensen, E. J. M., van der Meer, Y., van Veen, J. A. R., Niemantsverdriet, J. W., 2007. Insight into the formation of the active phases in supported NiW hydrotreating catalysts. Applied Catalysis A: General 322, 16-32. DOI:10.1016/j.apcata.2007.01.003

Hinnemann, B., Nørskov, J. K., Topsøe, H., 2005. A Density Functional Study of the Chemical Differences between Type I and Type II $\mathrm{MoS}_{2}$-Based Structures in Hydrotreating Catalysts. Journal of Physical Chemistry B 109, 2245-2253.

DOI:10.1021/jp048842y

Ho, T. C., 2004. Deep HDS of diesel fuel: chemistry and catalysis. Catalysis Today $98,3-8$ DOI:10.1016/j.cattod.2004.07.048

Lizama, L. Y., 2009. Desarrollo de catalizadores de hidrodesulfuración preparados a partir de heteropoliácidos soportados en SBA-15 modificado con $\mathrm{Al}, \mathrm{Zr}$ y Ti, Tesis doctoral. Universidad Nacional Autónoma de México, México.

Mogica-Betancourt, J. C., López-Benítez, A., Montiel-López, J. R., Massin, L., Aouine, M., Vrinat, M., Berhault, G., Guevara-Lara, A., 2014. Interaction effects of nickel polyoxotungstate with the $\mathrm{Al}_{2} \mathrm{O}_{3}-\mathrm{MgO}$ support for application in dibenzothiophene hydrodesulfurization. Journal of Catalysis 313, 9-23.

DOI:10.1016/j.jcat.2014.02.009 
Nag, N. K., Sapre, A. V., Broderick, D. H., Gates, B. C., 1979. Hydrodesulfurization of polycyclic aromatics catalyzed by sulfided $\mathrm{CoO}$ $\mathrm{MoO}_{3} / \gamma-\mathrm{Al}_{2} \mathrm{O}_{3}$ : The relative reactivities. Journal of Catalysis 57, 509-512. DOI:10.1016/0021-9517(79)90016-2

Puigcerver, M., Carrascal, M. D., 2008. El medio atmosférico: meteorología y contaminación. UBE, Barcelona.

Rangarajan, S., Mavrikakis, M., 2017. On the Preferred Active Sites of Promoted $\mathrm{MoS}_{2}$ for Hydrodesulfurization with Minimal Organonitrogen Inhibition. American Chemical Society Catalysis 7(1), 501-509. DOI:10.1021/acscatal.6b02735

Scheffer, B., Mangnus, P. J., Moulijn, J. A., 1990. A TemperatureProgrammed Sulfiding Study of NiO- $\mathrm{WO}_{3} / \mathrm{Al}_{2} \mathrm{O}_{3}$ Catalysts. Journal of Catalysis 121(1), 18-30. DOI:10.1016/0021-9517(90)90213-4

Shriver, D. F., Atkins, P. W., Langford, C. H., 2004. Química Inorgánica. Reverté S.A, Barcelona.

Stanislaus, A., Cooper, B. H., 1994. Aromatic Hydrogenation Catalysis: A Review. Catalysis Reviews-Science and Engineering 36(1), 75-123. DOI: 10.1080/01614949408013921

Topsøe, H., Clausen, B. S., Massoth, F. E., 1996. Hydrotreating Catalysis. Springer-Verlag Berlín Heidelberg, Alemania.

Topsøe, H., 2007. The role of Co-Mo-S type structures in hydrotreating catalysts. Applied Catalysis 322, 3-8. DOI:10.1016/j.apcata.2007.01.002

Vatutina, Y. V., Klimov, O. V., Stolyarova, E. A., Nadeina, K. A., Danilova, I. G., Chesalov, Y. A., Gerasimov, E. Y., Prosvirin, I. P., Noskov, A. S., 2019. Influence of the phosphorus addition ways on properties of CoMocatalysts of hydrotreating. Catalysis Today 329, 13-23. DOI: 10.1016/j.cattod.2019.01.005

Vissenberg, M. J., van der Meer, Y., Hensen, E. J. M., de Beer, V. H. J., van der Kraan, A. M., van Santen, R. A., van Veen, J. A. R., 2001. The Effect of Support Interaction on the Sulfidability of $\mathrm{Al}_{2} \mathrm{O}_{3}$ - and $\mathrm{TiO}_{2}-$ Supported CoW and NiW Hydrodesulfurization Catalysts. Journal of Catalysis 198, $151-163$

DOI:10.1006/jcat.2000.3132

Wilson, M. F., Fischer, I. P., Kriz, J. F., 1985. Hydrogenation of aromatic compounds in synthetic crude distillates catalyzed by sulfide $\mathrm{Ni}-\mathrm{W} / \gamma$ $\mathrm{Al}_{2} \mathrm{O}_{3}$. Journal of Catalysis 95, 155-156.

DOI: 10.1016/0021-9517(85)90016-8

Wivel, C., Candia, R., Clausen, B.S., Mørup, S., Topsøe, H., 1981. On the Catalytic Significance of a Co-Mo-S Phase in Co-Mo/ $\mathrm{Al}_{2} \mathrm{O}_{3}$ Hydrodesulfurization Catalysts: Combined in Situ Mössbauer Emission Spectroscopy and Activity Studies. Journal of Catalysis 68, 433-453. DOI:10.1016/0021-9517(81)90115-9

Zhang, L., Dong, P., Fu, W., Dai, Q., Tang, T., 2018. MOR nanosheet assemblies supported NiW catalysts in the hydrodesulfurization of the 4,6 DMDBT: Effect of $\mathrm{Ni} / \mathrm{W}$ ratio on the catalytic performance. Catalysis Today 316, 99-106. DOI:10.1016/j.cattod.2018.01.032

Zuo, D., Li, D., Nie, H., Shi, Y., Lacroix, M., Vrinat, M., 2004a. Acid-base properties of $\mathrm{NiW} / \mathrm{Al}_{2} \mathrm{O}_{3}$ sulfided catalysts: relationship with hydrogenation, isomerization and hydrodesulfurization reactions. Journal of Molecular Catalysis A: Chemical 211, 179-189. DOI: 10.1016/j.molcata.2003.10.018

Zuo, D., Vrinat, M., Nie, H., Maugé, F., Shi, Y., Lacroix, M., Li, D., 2004b. The formation of the active phases in sulfided $\mathrm{NiW} / \mathrm{Al}_{2} \mathrm{O}_{3}$ catalysts and their evolution during post-reduction treatment. Catalysis Today 93-95, 751-760

DOI:10.1016/j.cattod.2004.06.078 